\title{
Fully endoscopic lumbar interbody fusion using a percutaneous unilateral biportal endoscopic technique: technical note and preliminary clinical results
}

\author{
*Dong Hwa Heo, MD, PhD, ${ }^{1}$ Sang Kyu Son, MD, ${ }^{2}$ Jin Hwa Eum, MD, ${ }^{3}$ and \\ Choon Keun Park, MD, PhD, ${ }^{1}$ on behalf of the International Unilateral Biportal Endoscopic \\ Spine Surgery Research Society
}

${ }^{1}$ Department of Neurosurgery, Spine Center, The Leon Wiltse Memorial Hospital, Suwon; ${ }^{2}$ Department of Neurosurgery, Spine Center, Gangdong Hospital, Busan; and '3epartment of Neurosurgery, Spine Center, Centum Hospital, Changwon, Korea

\begin{abstract}
OBJECTIVE Minimally invasive spine surgery can minimize damage to normal anatomical structures. Recently, fully endoscopic spine surgeries have been attempted for lumbar fusion surgery. In this study, the authors performed a percutaneous unilateral biportal endoscopic (UBE) technique as a minimally invasive surgery for lumbar fusion. The purpose of this study is to present the UBE technique of fully endoscopic lumbar interbody fusion (LIF) and to analyze the clinical results.

METHODS Patients who were to undergo single-level fusion surgery from L3-4 to L5-S1 were enrolled. Two channels (endoscopic portal and working portal) were used for endoscopic lumbar fusion surgery. All patients underwent follow-up for more than 12 months. Demographic characteristics, diagnosis, operative time, and estimated blood loss were evaluated. MRI was performed on postoperative Day 2. Clinical evaluations (visual analog scale [VAS] for the leg and Oswestry Disability Index [ODI] scores) were performed preoperatively and during the follow-up period.

RESULTS A total of 69 patients ( 24 men and 45 women) were enrolled in this study. The mean follow-up period was 13.5 months. Postoperative MRI revealed optimal direct neural decompression after fully endoscopic fusion surgery. VAS and ODI scores significantly improved after the surgery. There was no postoperative neurological deterioration.

CONCLUSIONS Fully endoscopic LIF using the UBE technique may represent an alternative minimally invasive LIF surgery for the treatment of degenerative lumbar disease. Long-term follow-up and larger clinical studies are needed to validate the clinical and radiological results of this surgery.

https://thejns.org/doi/abs/10.3171/2017.5.FOCUS17146
\end{abstract}

KEY WORDS lumbar; fusion surgery; endoscopy; minimally invasive surgery

$\mathrm{L}$ UMBAR fusion surgery is a treatment option for lumbar degenerative disease, such as segmental instability. 4,6,9,13,14 There are many types of lumbar fusion surgery, including anterior lumbar interbody fusion (ALIF), lateral lumbar interbody fusion (LLIF), posterior lumbar interbody fusion (PLIF), and transforaminal lumbar interbody fusion (TLIF).,4,9,11 Among these treatment modalities, PLIF and TLIF can result in direct posterior neural decompression..$^{11}$ Although conventional posterior open fusion surgeries can achieve wide decompression of neural structures and provide stabilization for surgically treated segments, they can also result in extensive destruction of posterior anatomical structures, thereby requiring long periods of recovery. Therefore, various types of minimally invasive spine surgeries have been attempted to treat lumbar degenerative disease. . $^{6,11,12,15,17}$ These surgeries can minimize injury to normal anatomical structures during the procedures. ${ }^{1,3,5,7,10}$ Recently, spinal endoscopic surgeries have been attempted in the field of lumbar decompression and fusion surgery. 2,3,7,10,17

We have been using the percutaneous unilateral biportal endoscopic (UBE) technique for lumbar discectomy,

ABBREVIATIONS ALIF = anterior lumbar interbody fusion; LLIF = lateral LIF; ODI = Oswestry Disability Index; TLIF = transforaminal LIF; UBE = unilateral biportal endoscopic; VAS = visual analog scale.

SUBMITTED March 4, 2017. ACCEPTED May 22, 2017.

INCLUDE WHEN CITING DOI: 10.3171/2017.5.FOCUS17146.

${ }^{*}$ Drs. Heo and Son contributed equally to this work. 

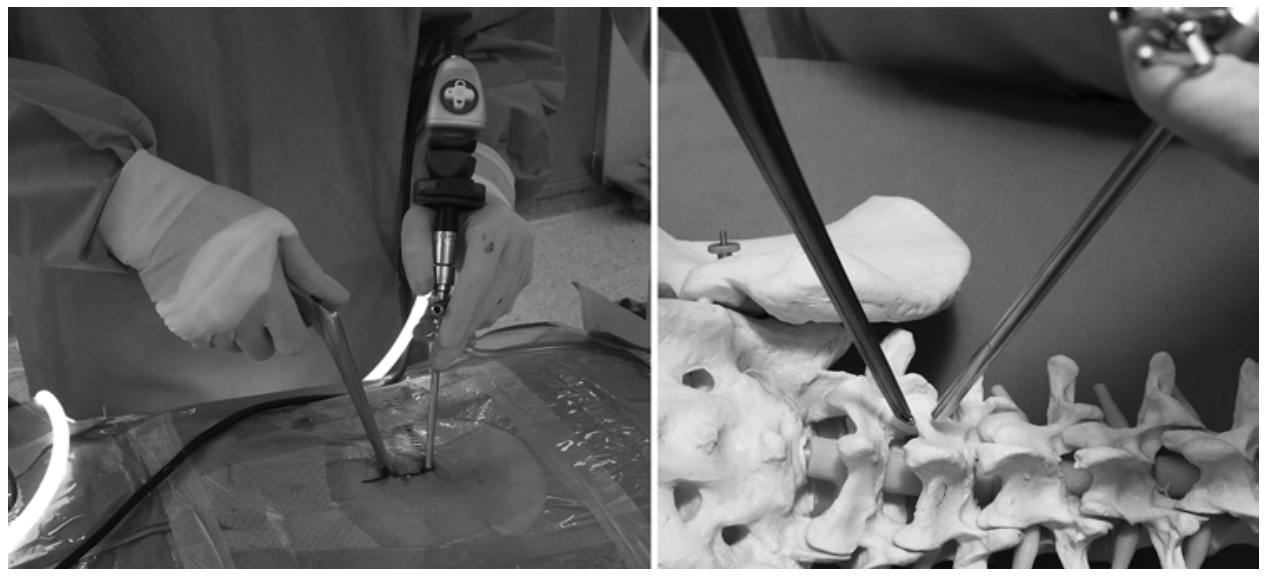

FIG. 1. Overview of the percutaneous UBE surgery in the spine.

decompressive laminectomy, and lateral foraminotomy (Fig. 1). ${ }^{3,7}$ We have also used the biportal endoscopic technique in minimally invasive lumbar fusion surgeries, such as TLIF. The purpose of this study was to present the surgical technique of fully endoscopic LIF using the UBE technique and to analyze the clinical results.

\section{Methods}

All contributing authors have extensive experience in percutaneous UBE surgeries, such as discectomy for lumbar disc herniation, simple decompression for lumbar stenosis, and lateral foraminotomy for lumbar foraminal and extraforaminal lesions. Before clinical application of this fully endoscopic LIF, we practiced fully endoscopic TLIF using the UBE technique at 15 lumbar levels in 5 cadavers. We prospectively started performing endoscopic LIF using the UBE technique in 2014.

\section{Indication of Endoscopic Lumbar Interbody Fusion}

We initially only performed single-level fusion surgery from L3-4 to L5-S1. Indications of minimally invasive endoscopic LIF surgery using UBE were the same as those for TLIF, including degenerative spondylolisthesis, isthmic spondylolisthesis, spinal stenosis with instability, and central stenosis with concomitant foraminal stenosis, with complete spinal canal decompression. We did not perform endoscopic fusion in cases of infection, spondylodiscitis, vertebral fractures, or high-grade spondylolisthesis.

\section{Surgical Technique}

All operations were performed under general or epidural anesthesia; general endotracheal anesthesia was preferred. The patient was placed prone on a radiolucent table to enable the use of a C-arm fluoroscope for posterior fusion surgeries. Waterproof draping was usually used. A specialized drape was preferred for shoulder arthroscopy and the lumbar endoscopic procedure. Two holes were made for this operation. Two ipsilateral skin incisions were made in the paramedian area, appearing above and below a $1-\mathrm{cm}$ area at the midportion of the spinous process or disc space in the lateral projection and on the ipsi- lateral medial border of the pedicle in the anteroposterior projection. ${ }^{6}$

For left-sided approaches, the left hole acted as the endoscopic portal and the right hole was the working portal (Fig. 1). After making a small incision in the skin and fascia, serial dilators were inserted to make 2 portals under C-arm fluoroscopic guidance. A lamina was then dissected using a small specialized lamina dissector in the working portal under fluoroscopic guidance. ${ }^{6}$ Effort was made to generate 2 portals using the interfascicular area to minimize muscle damage. ${ }^{6}$ Finally, endoscopic irrigation systems were used, and the irrigation fluid was drained from the endoscopic portal to the working portal. The irrigation fluid drained naturally, without the aid of a retractor or tube. Additional lamina and facet joint dissection and bleeding control were performed using radiofrequency coagulators (Video 1).

VIDEO 1. Video clip demonstrating fully endoscopic LIF using the percutaneous UBE technique. First, we performed unilateral laminotomy with bilateral decompression of the operative segment under endoscopic visualization. An ipsilateral facetectomy was then performed. Finally, we prepared the endplate before performing complete discectomy. On confirmation of the completion of endplate preparation using endoscopic visualization, a long, straight cage was inserted after packing of fusion materials, such as allograft bone or autograft bone, into the interbody space. Inf. = inferior. Copyright Dong Hwa Heo. Published with permission. Click here to view.

Our endoscopic LIF is similar to minimally invasive TLIF using a tubular retractor. ${ }^{11}$ Surgical procedures were performed under a highly magnified endoscopic view. If endoscopic fusion was performed at L4-5, an ipsilateral partial hemilaminectomy was made with the aid of an endoscopic drill and Kerrison punch at this level (Fig. 2A). After ipsilateral decompression, the contralateral sublaminar area was decompressed by sublaminar drilling to remove the ligamentum flavum (Fig. 2B). Complete exposure of contralateral and ipsilateral descending nerve roots was confirmed. After complete decompression of the central canal, the ipsilateral facet joint was removed.

After unilateral facetectomy, the disc was totally removed using pituitary forceps and reamers. Epidural bleeding was controlled using radiofrequency coagula- 

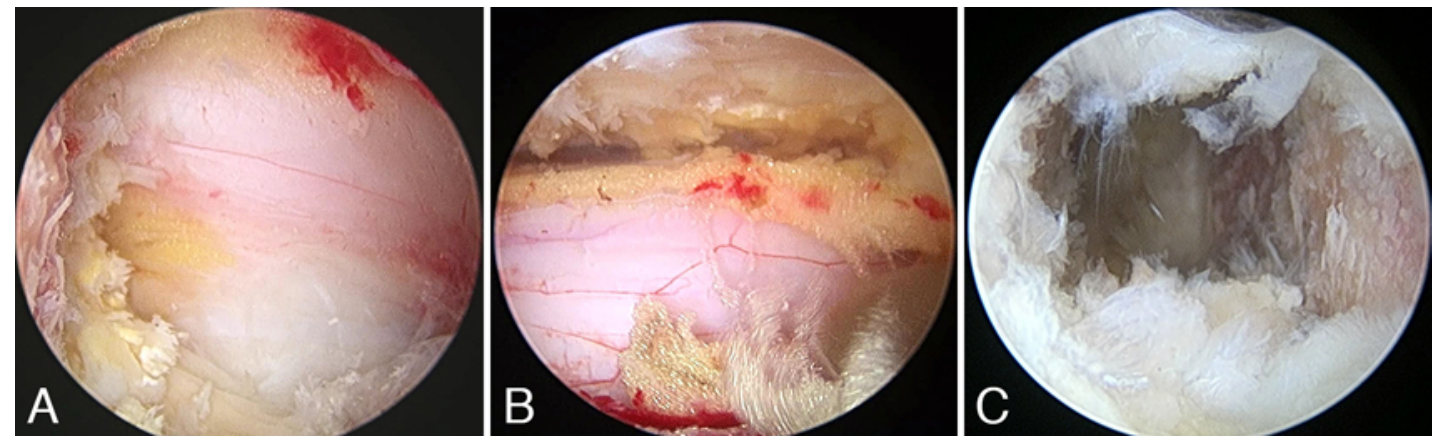

FIG. 2. Intraoperative endoscopic images obtained during endoscopic LIF, showing decompression of the central canal and ipsilateral facetectomy $(\mathbf{A})$, contralateral sublaminar decompression $(\mathbf{B})$, and endplate preparation after removing the vertebral cartilaginous endplate (C).

tors. Only the cartilaginous endplate was completely removed using ring curettes and endplate curettes. Endplate preparation was monitored with endoscopic visualization (Fig. 2C). Autologous bone chips from lamina and facet were impacted into the disc space using a specialized funnel (Fig. 3 left). Finally, a long, straight cage packed with autologous bone or fusion material was inserted after dura retraction under fluoroscopic guidance (Fig. 3 right). This cage was inserted deeper into the intervertebral space with the aid of an impactor.

Two ipsilateral percutaneous pedicle screws were inserted using the 2 previously described skin incisions. The 2 percutaneous pedicle screws were then contralaterally inserted after making 2 small new skin incisions (Figs. 4 and 5). A small drainage catheter was inserted to prevent postoperative epidural hematoma (Video 1).

\section{Analysis of Clinical Results}

We only enrolled patients who were to undergo singlelevel fusion. All patients underwent follow-up for more than 12 months. Demographic characteristics, diagnosis, operative time, and estimated blood loss were evaluated, as were surgical complications. MRI was performed on postoperative Day 2 to evaluate the optimal neural decompression status (Fig. 6). Clinical findings were evaluated in the preoperative and postoperative periods using the visual analog scale (VAS) for the legs and Oswestry Disability Index (ODI).

We performed this investigation in accordance with our institutional guidelines, which comply with international laws and policies (institutional review board of The Leon Wiltse Memorial Hospital). Statistical analysis was performed using the Wilcoxon rank-sum test; $\mathrm{p}<0.05$ was considered statistically significant. We used R 3.1.2 for Windows for the statistical analysis.

\section{Results}

A total of 69 patients ( 24 men and 45 women; mean age [ \pm SD] $71.2 \pm 7.8$ years) have been enrolled in our study since March 2014. The mean follow-up period was 13.5 \pm 7.1 months. A total of 69 vertebral levels in 69 patients were treated using fully endoscopic interbody fusion; 51 patients had degenerative spondylolisthesis, 9 patients had isthmic spondylolisthesis, 6 patients had central stenosis with segmental instability, and 3 patients had central stenosis with concomitant foraminal stenosis. The operative levels ranged from L3-4 to L5-S1: L3-4 in 9 patients, L4-5 in 48 patients, and L5-S1 in 12 patients (Table 1).

Postoperative MRI revealed optimal neural decompression of the treated segments in all patients (Fig. 6). Preoperative VAS and ODI scores improved significantly after surgery: VAS scores from $8.12 \pm 0.63$ preoperatively to $2.79 \pm 1.24$ at the last follow-up visit $(\mathrm{p}<0.05)$ and ODI scores from $45.65 \pm 12.97$ to $15.41 \pm 9.07(\mathrm{p}<0.05)$. No patient experienced neurological deterioration after surgery. The mean estimated blood loss, including drainage blood, was $85.5 \pm 19.41 \mathrm{ml}$. The mean operative time was $165.8 \pm 25.5$ minutes.

We observed 5 cases of perioperative complications: dural tear in 2 patients and postoperative epidural hematoma in 3 patients. None of these patients required revision surgery and their complications spontaneously resolved with conservative management. Revision surgery was not required in any patient during the follow-up period.

\section{Discussion}

Although conventional open posterior fusion surgery is an effective treatment for lumbar degenerative disease, surgical destruction of muscle and ligamentous structures
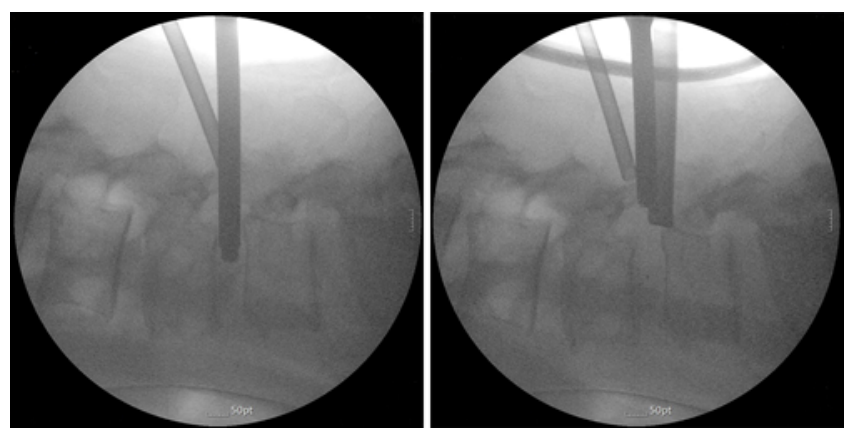

FIG. 3. Intraoperative radiographs. Left: After endplate preparation, chip bone and allograft bone are impacted into the disc space using a funnel. Right: A long cage is inserted percutaneously under fluoroscopic guidance. 

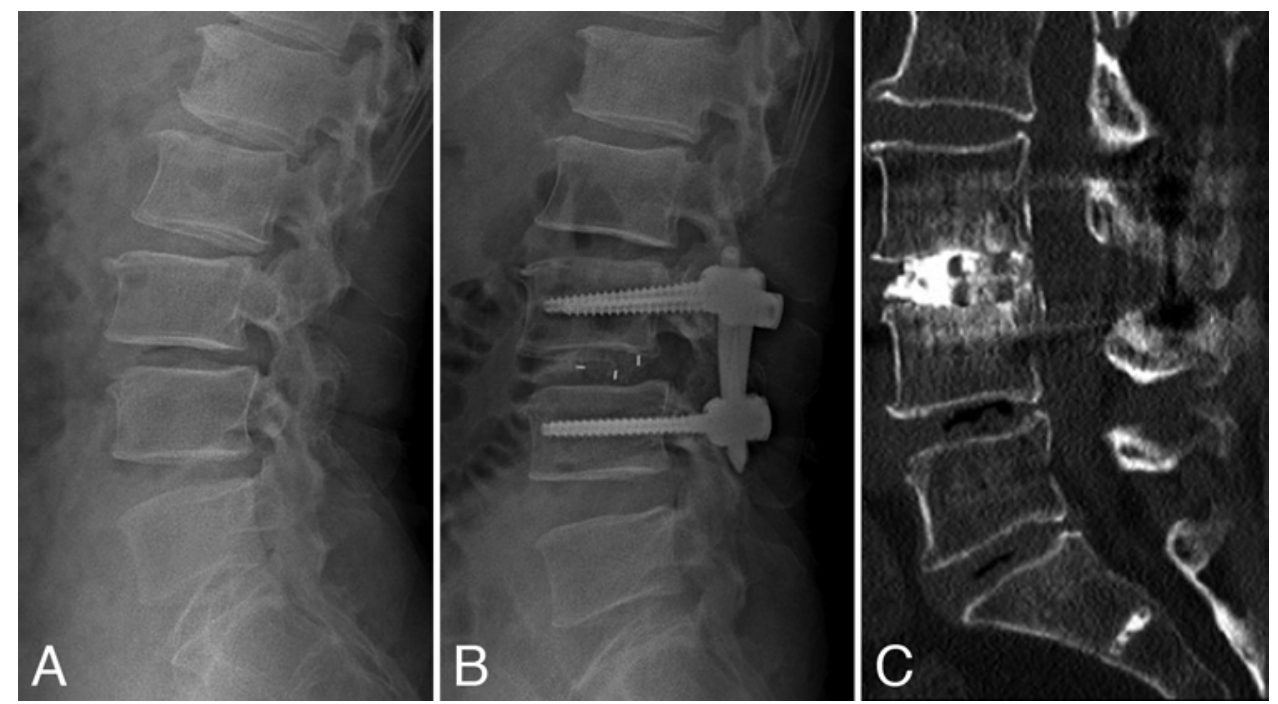

FIG. 4. A: Preoperative lateral radiograph showing degenerative spondylolisthesis with disc space narrowing at L3-4. B: Postoperative lateral simple radiograph after fully endoscopic LIF. C: Sagittal CT image obtained 14 months postoperatively, showing interbody fusion.

can lead to postoperative back pain and muscle atrophy. ${ }^{6,11}$ Therefore, more time may be required for functional recovery before returning to work after conventional posterior open fusion surgery. As a result, minimally invasive fusion techniques have been developed to minimize injuries to posterior musculo-ligamentous structures. ${ }^{6,7,10,12,17}$

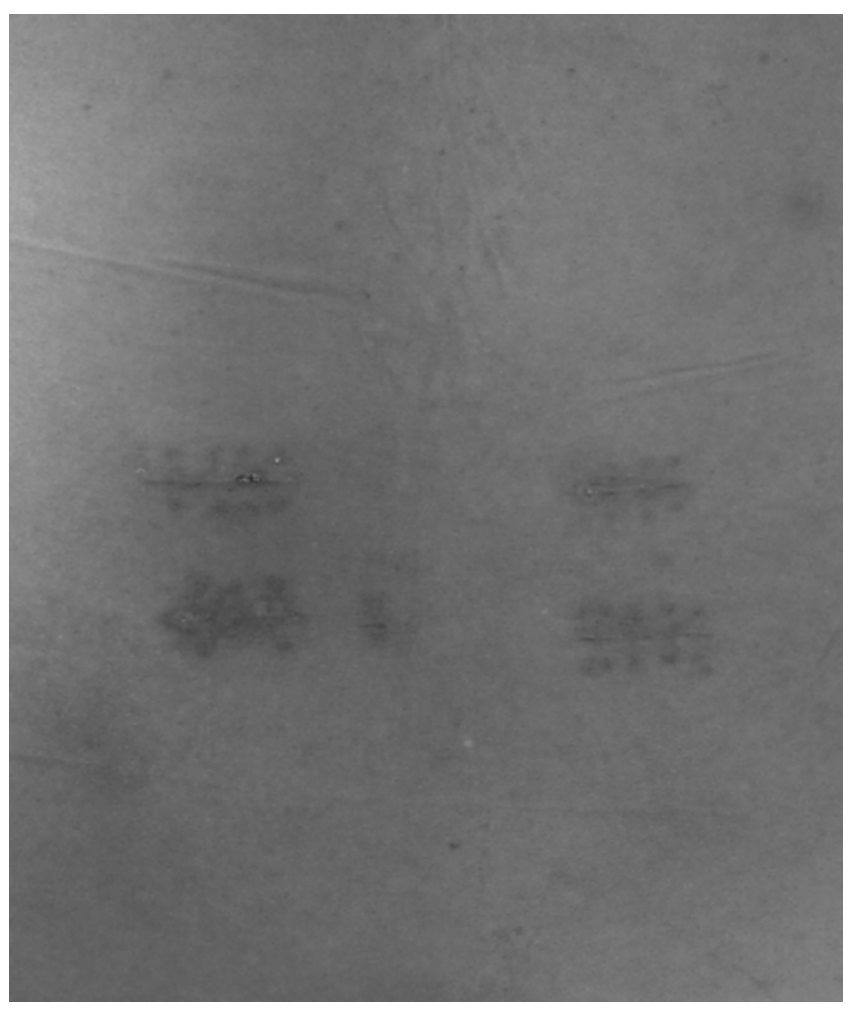

FIG. 5. Skin incision points for the fully endoscopic LIF using the biportal endoscopic system.
LLIF and ALIF are both good surgical treatment options. They can preserve posterior structures, such as laminae, ligaments, and muscles. Although lateral and anterior approaches have indirect decompressive effects, such as disc height restoration, spondylolisthesis reduction, and a foramen-widening effect, these approaches have limited indications due to the impossibility of achieving direct posterior decompression. ${ }^{6,8,9}$ In contrast, minimally invasive TLIF using a tubular retractor can achieve direct decompression through discectomy, facetectomy, and bilateral laminoforaminotomy via a unilateral approach. ${ }^{5,11,16} \mathrm{In}$ the present study, we tested minimally invasive TLIF using the biportal endoscopic approach to achieve maximal preservation of normal musculo-ligamentous structures rather than using tubular retractors. The percutaneous UBE approach combines the advantages of standard open surgery and endoscopic spinal surgery. This technique is a modification of the endoscopic interlaminar approach. ${ }^{2,6}$ The percutaneous UBE approach is similar to joint arthroscopic surgeries and video-assisted thoracoscopic surgeries. The UBE approach uses 2 different channels (or "portals") as in joint arthroplasty (Fig. 1). Two unilateral skin entry points are made ipsilaterally. One portal is used for the endoscope, while the other is used for the entry of surgical instruments. ${ }^{3,6}$ Compared with the 1-portal lumbar endoscopic approach, the working portal is used only for surgical instruments. Therefore, the handling and movement of instruments is unrestricted and convenient. In addition, standard spine instruments can be inserted and used through the working portal. The joint arthroscopic shaver drill and short interlaminar endoscopic drill system are of particular usefulness in the UBE approach. Moreover, the endoscopic view is similar to that of posterior microscopic surgery. The UBE approach allows the surgical area to be viewed at high magnification, and its endoscopic view is familiar to spine surgeons. ${ }^{7}$

Endplate preparation is important for interbody fusion. 

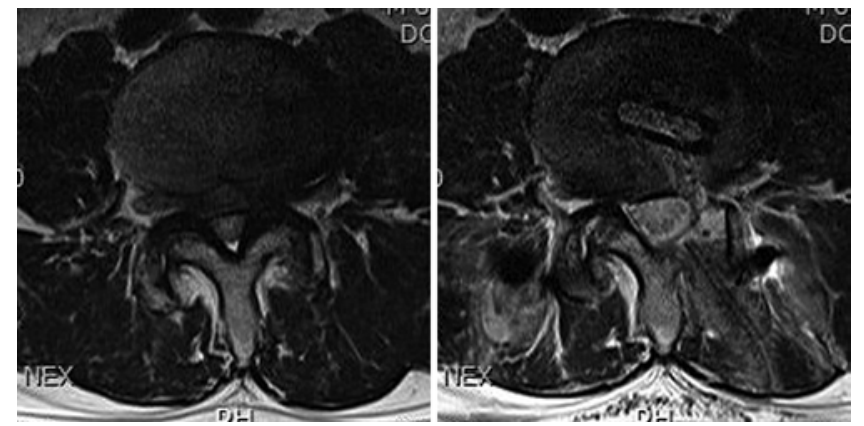

FIG. 6. Left: Preoperative axial MR image showing lumbar canal stenosis. Right: Axial MR image showing that spinal stenosis was completely decompressed after fully endoscopic fusion surgery.

Complete endplate preparation might be difficult due to the blind nature of the LLIF approach and other endoscopic approaches. ${ }^{6}$ Conventional endplate reamers and curettes are available, as with open surgery, for use in the UBE approach. In addition, we were able to confirm completion of endplate preparation using the endoscopic view (Fig. 2C). We sometimes performed endplate preparation again after endoscopic exploration of the disc space due to incomplete removal of the cartilaginous endplate. In these cases, we inserted autograft bone from lamina and inferior articular processes and allograft bone into the disc space before cage insertion. Because continuous saline irrigation may interfere with intradiscal bone packing, a specialized intradiscal funnel is necessary for bone packing into the disc space (Fig. 3). Since this operation can minimize intraoperative damage to posterior ligamentomuscular structures, postoperative back pain might also be minimized.

Fully endoscopic fusion surgery has some limitations regarding direct reduction of spondylolisthesis. A reduction in spondylolisthesis occurred spontaneously during the operation after facetectomy and discectomy. Recently, we have attempted to reduce the degree of spondylolisthesis incurred using a percutaneous reduction pedicle screw system. We suggest that high-grade spondylolisthesis is a contraindication for endoscopic fusion surgery.

Perioperative complications consisted of dural tears (n $=2$ ) and epidural hematomas $(\mathrm{n}=3)$. A small durotomy occurred during the removal of the ligamentum flavum. If a dural tear cannot be repaired during UBE surgery, conservative management, such as bed rest, could spontaneously resolve the tear. ${ }^{7}$ However, if a large dural tear or defect were to occur, endoscopic surgery should be converted to open microscopic surgery for direct dura repair. Recently, we have tried to repair durotomy areas directly using small dura clips and sealing material (fibrin collagen patch). Regarding hematoma formation, small amounts of epidural bleeding might be overlooked because of continuous drainage of saline irrigation, which results in pressure. Therefore, postoperative epidural hematoma can occur. Meticulous epidural bleeding and drainage catheter insertion should be performed to prevent hematoma formation.

The percutaneous UBE procedure is fundamentally different from that of 1-portal spinal endoscopic surgery. The 1-portal endoscopic system uses working sleeve de-
TABLE 1. Patient characteristics

\begin{tabular}{lc}
\hline \multicolumn{1}{c}{ Characteristic } & Value \\
\hline Mean age in yrs & $71.2 \pm 7.8$ \\
\hline Sex & 24 \\
\hline M & 45 \\
\hline F & $13.5 \pm 7.1$ \\
\hline Mean follow-up period in mos & 9 \\
\hline Level treated & 48 \\
\hline L3-4 & 12 \\
\hline L4-5 & 51 \\
\hline L5-S1 & 9 \\
\hline Diagnosis & 6 \\
\hline Degenerative spondylolisthesis & 3 \\
\hline Isthmic spondylolisthesis & $85.5 \pm 19.4$ \\
\hline Central stenosis w/ segmental instability & $165.8 \pm 25.5$ \\
\hline Central stenosis w/ concomitant foraminal stenosis \\
\hline Mean estimated blood loss in ml \\
\hline Mean operative time in mins & 2 \\
\hline Postop complications & 3 \\
\hline Dural tear & \\
\hline Postop hematoma & \\
\hline
\end{tabular}

Values are presented as the number of patients unless stated otherwise.

vices for soft-tissue retraction; that is, a single portal (the working sleeve) is used for both the endoscope and the surgical instruments. In contrast, the biportal endoscopic system does not use a working sleeve. A scope and instrument are needed to work in the cavity filled with irrigation fluid. Initially, this cavity was made between the interfascicular area, interlaminar space, and lamina. As the size of this cavity increases due to exposure of epidural space via laminectomy and flavectomy, visualization through the endoscope becomes clearer.

In UBE surgery, because the working portal is used only as the entrance for the spinal instruments, and not the scope, various spinal instruments and endoscopic devices are available. Another benefit of UBE surgery is that, if there is an increase in the rate at which specialized instruments for UBE surgery are developed, percutaneous UBE surgery might be performed more easily and conveniently.

The percutaneous UBE procedure, as with 1-portal fully endoscopic surgeries, has a short learning curve for the surgeon. Gaining experience in many cases of microsurgery using a tubular retractor is recommended before attempting biportal endoscopic surgery. In addition, discectomy or decompressive laminotomy performed via UBE surgery should be started before endoscopic fusion surgery is performed.

In this paper, we present the preliminary results of endoscopic fusion with the UBE technique. The results of this study cannot be extrapolated. To improve the assessment of clinical outcomes after UBE surgery, a larger number of patients should be studied and undergo followup. In addition, a comparative study including other types of fusion surgery should be performed in the future. 


\section{Conclusions}

The surgical endoscopic view of the percutaneous UBE technique is similar to that of minimally invasive surgery using tubular retractors. This technique can achieve direct neural decompression similar to conventional open surgery. Our results suggest that fully endoscopic LIF using the percutaneous UBE method might be an alternative to minimally invasive LIF surgery for treating degenerative lumbar disease. However, long-term follow-up and larger clinical studies are needed to validate the clinical and radiological results of this surgery.

\section{References}

1. Amin BY, Tu TH, Mummaneni PV: Mini-open transforaminal lumbar interbody fusion. Neurosurg Focus 35 Suppl:Video 2, 2013

2. De Antoni DJ, Claro ML, Poehling GG, Hughes SS: Translaminar lumbar epidural endoscopy: anatomy, technique, and indications. Arthroscopy 12:330-334, 1996

3. Eun SS, Eum JH, Lee SH, Sabal LA: Biportal endoscopic lumbar decompression for lumbar disk herniation and spinal canal stenosis: a technical note. J Neurol Surg A Cent Eur Neurosurg 78:390-396, 2017

4. Guan J, Bisson EF, Dailey AT, Hood RS, Schmidt MH: Comparison of clinical outcomes in the National Neurosurgery Quality and Outcomes Database for open versus minimally invasive transforaminal lumbar interbody fusion. Spine (Phila Pa 1976) 41:E416-E421, 2016

5. Hari A, Krishna M, Rajagandhi S, Rajakumar DV: Minimally invasive transforaminal lumbar interbody fusion-indications and clinical experience. Neurol India 64:444-454, 2016

6. Heo DH, Choi WS, Park CK, Kim JS: Minimally invasive oblique lumbar interbody fusion with spinal endoscope assistance: technical note. World Neurosurg 96:530-536, 2016

7. Hwa Eum J, Hwa Heo D, Son SK, Park CK: Percutaneous biportal endoscopic decompression for lumbar spinal stenosis: a technical note and preliminary clinical results. J Neurosurg Spine 24:602-607, 2016

8. Kepler CK, Sharma AK, Huang RC, Meredith DS, Girardi FP, Cammisa FP Jr, et al: Indirect foraminal decompression after lateral transpsoas interbody fusion. J Neurosurg Spine 16:329-333, 2012

9. Kim JS, Choi WG, Lee SH: Minimally invasive anterior lumbar interbody fusion followed by percutaneous pedicle screw fixation for isthmic spondylolisthesis: minimum 5-year follow-up. Spine J 10:404-409, 2010

10. Komp M, Hahn P, Oezdemir S, Giannakopoulos A, Heikenfeld R, Kasch R, et al: Bilateral spinal decompression of lumbar central stenosis with the full-endoscopic interlaminar versus microsurgical laminotomy technique: a prospective, randomized, controlled study. Pain Physician 18:61-70, 2015

11. Lee CK, Park JY, Zhang HY: Minimally invasive transfo- raminal lumbar interbody fusion using a single interbody cage and a tubular retraction system: technical tips, and perioperative, radiologic and clinical outcomes. J Korean Neurosurg Soc 48:219-224, 2010

12. Minamide A, Yoshida M, Yamada H, Nakagawa Y, Kawai M, Maio K, et al: Endoscope-assisted spinal decompression surgery for lumbar spinal stenosis. J Neurosurg Spine 19:664-671, 2013

13. Mummaneni PV, Dhall SS, Eck JC, Groff MW, Ghogawala Z, Watters WC III, et al: Guideline update for the performance of fusion procedures for degenerative disease of the lumbar spine. Part 11: interbody techniques for lumbar fusion. J Neurosurg Spine 21:67-74, 2014

14. Resnick DK, Watters WC III, Mummaneni PV, Dailey AT, Choudhri TF, Eck JC, et al: Guideline update for the performance of fusion procedures for degenerative disease of the lumbar spine. Part 10: lumbar fusion for stenosis without spondylolisthesis. J Neurosurg Spine 21:62-66, 2014

15. Than KD, Park P, Fu KM, Nguyen S, Wang MY, Chou D, et al: Clinical and radiographic parameters associated with best versus worst clinical outcomes in minimally invasive spinal deformity surgery. J Neurosurg Spine 25:21-25, 2016

16. Vazan M, Gempt J, Meyer B, Buchmann N, Ryang YM: Minimally invasive transforaminal lumbar interbody fusion versus open transforaminal lumbar interbody fusion: a technical description and review of the literature. Acta Neurochir (Wien) 159:1137-1146, 2017

17. Wang MY, Grossman J: Endoscopic minimally invasive transforaminal interbody fusion without general anesthesia: initial clinical experience with 1-year follow-up. Neurosurg Focus 40(2):E13, 2016

\section{Disclosures}

The authors report no conflict of interest concerning the materials or methods used in this study or the findings specified in this paper.

\section{Author Contributions}

Conception and design: all authors. Acquisition of data: Heo, Son. Analysis and interpretation of data: Heo, Son. Drafting the article: Heo, Son. Critically revising the article: Son. Reviewed submitted version of manuscript: Park, Heo, Son. Study supervision: Park, Son, Eum.

\section{Supplemental Information \\ Videos \\ Video 1. https://vimeo.com/221727236.}

\section{Correspondence}

Choon Keun Park, Department of Neurosurgery, Spine Center, The Leon Wiltse Memorial Hospital, 994-3 Ingye-Dong, PaldalGu, Suwon 442-833, Republic of South Korea. email: allspine@ gmail.com. 